\title{
Enhancing erbium emission by strain engineering in GaN heteroepitaxial layers
}

\author{
I. W. Feng, ${ }^{1}$ J. Li, ${ }^{1}$ A. Sedhain, ${ }^{1}$ J. Y. Lin, ${ }^{1}$ H. X. Jiang, ${ }^{1, a)}$ and J. Zavada ${ }^{2}$ \\ ${ }^{1}$ Department of Electrical and Computer Engineering, Texas Tech University, Lubbock, Texas 79409, USA \\ ${ }^{2}$ Department of Electrical and Computer Engineering, North Carolina State University, Raleigh, \\ North Carolina 27695-7911, USA
}

(Received 16 December 2009; accepted 28 December 2009; published online 21 January 2010)

\begin{abstract}
Much research has been devoted to the incorporation of erbium (Er) into semiconductors aimed at achieving photonic integrated circuits with multiple functionalities. GaN appears to be an excellent host material for Er ions due to its structural and thermal stability. Er-doped GaN (GaN:Er) epilayers were grown on different templates, $\mathrm{GaN} / \mathrm{Al}_{2} \mathrm{O}_{3}, \mathrm{AlN} / \mathrm{Al}_{2} \mathrm{O}_{3}, \mathrm{GaN} / \mathrm{Si}$ (111), and c-GaN bulk. The effects of stress on $1.54 \mu \mathrm{m}$ emission intensity, caused by lattice mismatch between the GaN:Er epilayer and the substrate, were probed. The emission intensity at $1.54 \mu \mathrm{m}$ increased with greater tensile stress in the c-direction of the GaN:Er epilayers. These results indicate that the characteristics of photonic devices based on GaN:Er can be optimized through strain engineering. (C) 2010 American Institute of Physics. [doi:10.1063/1.3295705]
\end{abstract}

Rare earth (RE) doped semiconductors have been studied extensively due to their intra $4 f$-electron transitions with emission wavelengths covering from visible to infrared region. ${ }^{1}$ Trivalent erbium $\left(\mathrm{Er}^{3+}\right)$ has been of particular interest since transitions from its first excited state $\left({ }^{4} \mathrm{I}_{13 / 2}\right)$ to the ground state $\left({ }^{4} \mathrm{I}_{15 / 2}\right)$ provides $1.54 \mu \mathrm{m}$ emission, which matches the minimum loss band of silica fiber. ${ }^{1-3}$ This affords the possible applications of Er doped semiconductors for emitters and optical waveguide amplifiers at the optical communication wavelength. Extensive studies have been reported on Er incorporation into various kinds of semiconductor hosts, such as $\mathrm{Si}, \mathrm{SiGe}, \mathrm{GaAs}$, InP etc. ${ }^{4-8}$ However, the thermal quenching effect of $1.54 \mu \mathrm{m}$ emission intensity is severe for narrow band gap host materials ${ }^{9}$ and has impeded development for room temperature (RT) applications. In the last decade, special attention was given to Er doped IIInitride semiconductors since the thermal quenching of $1.54 \mu \mathrm{m}$ emission intensity is greatly reduced in these materials. ${ }^{2,9}$ Different methods have been employed to synthesize $\mathrm{Er}$ doped $\mathrm{GaN}$ (GaN:Er), including ion-implantation, ${ }^{10,11}$ molecular beam epitaxy, ${ }^{1,12,13}$ hydride vapor phase epitaxy, ${ }^{14}$ and metal-organic chemical vapor deposition (MOCVD). ${ }^{2}$ In our previous works, ${ }^{15-17}$ we demonstrated in situ growth of GaN:Er and the related applications of light emitting diodes and erbium doped nitride amplifiers. While most of the studies of Er doped GaN have been focused on the optical properties and crystalline quality, little attention has been given to the effects of stress on $1.54 \mu \mathrm{m}$ emission intensity, induced by lattice mismatch between the GaN:Er epilayer and the substrate. Since stress can be engineered during growth, the effect of strain on $\mathrm{GaN}: \mathrm{Er}$ may provide another way to enhance $1.54 \mu \mathrm{m}$ emission intensity and the characteristics of photonic devices based on $\mathrm{GaN}$ :Er. Here we report on the MOCVD growth of GaN:Er on various substrates and the resulting optical characteristics, crystalline quality, and surface morphology.

The GaN:Er thin layers were grown simultaneously by MOCVD on four different types of templates: $\mathrm{AlN} / \mathrm{Al}_{2} \mathrm{O}_{3}$,

${ }^{a)}$ Electronic mail: hx.jiang@ttu.edu.
$\mathrm{GaN} / \mathrm{Al}_{2} \mathrm{O}_{3}, \mathrm{GaN} / \mathrm{Si}$ (111), and c-GaN bulk. An undoped $\mathrm{GaN}$ thin layer was first grown on the substrates, and followed by growth of a GaN:Er epilayer with thickness $\sim 1 \mu \mathrm{m}$. The growth temperature was $1040{ }^{\circ} \mathrm{C}$. The details of the MOCVD growth conditions for GaN:Er epilayers can be found in our earlier reports. ${ }^{2,15}$ For optical analysis, photoluminescence (PL) spectra were taken at RT with above band gap excitation wavelength at $266 \mathrm{~nm}$. An InGaAs detector was used to measure the spectra near $1.54 \mu \mathrm{m} .^{2}$

As can be seen in Fig. 1, the GaN:Er film grown on $\mathrm{AlN} / \mathrm{Al}_{2} \mathrm{O}_{3}$ yields the strongest $1.54 \mu \mathrm{m}$ emission at $300 \mathrm{~K}$. The next strongest emission is from the GaN:Er film grown on $\mathrm{GaN} / \mathrm{Al}_{2} \mathrm{O}_{3}$, followed by that grown on the $\mathrm{GaN} / \mathrm{Si}$ (111) template. The PL intensities for GaN:Er films grown on these two templates are nearly comparable. The weakest emission was observed for GaN:Er film grown on the c-GaN bulk substrate. Intuitively, these results appear in contrary to the fact that the homoepilayer of $\mathrm{GaN}: \mathrm{Er}$ is expected to have the highest crystalline quality.

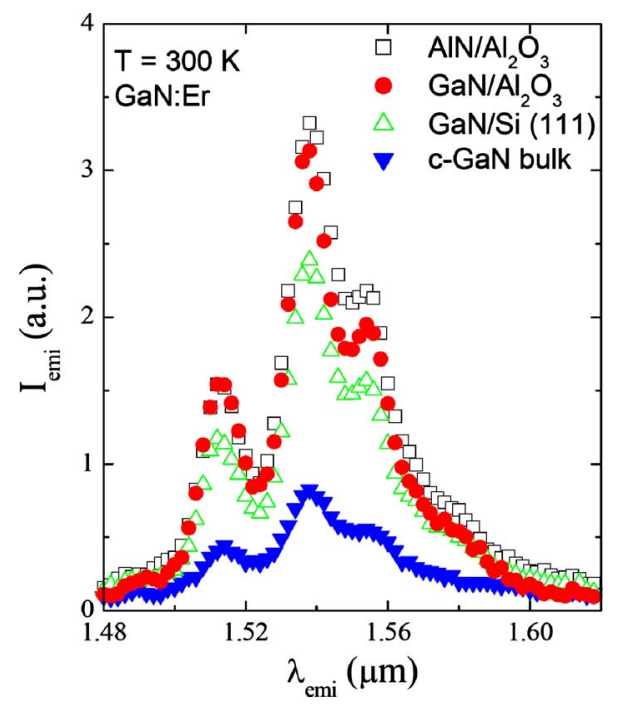

FIG. 1. (Color online) PL spectra near $1.54 \mu \mathrm{m}$ measured at $300 \mathrm{~K}$ for $\mathrm{GaN}$ :Er epilayers grown on different templates. 


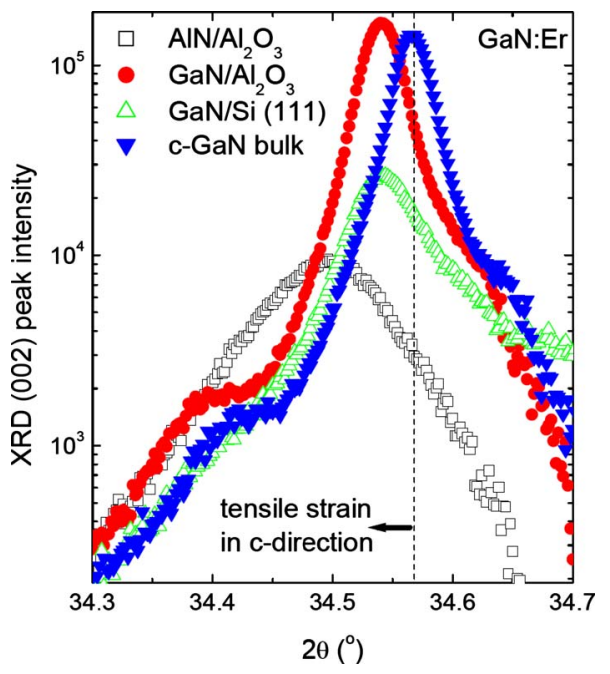

FIG. 2. (Color online) $\theta-2 \theta$ XRD spectra for GaN:Er layers grown on different templates. The diffraction peaks shifted to smaller angle relative to $2 \theta=34.57^{\circ}$ (c-GaN position).

In Fig. 2, the $\theta-2 \theta$ x-ray diffraction (XRD) spectra of (002) plane of the four samples are shown. It can be seen that use of different substrates gives rise to a shift in the diffraction peak relative to the peak position $\left(\sim 34.57^{\circ}\right)$ of the c-GaN reference. The lattice constant $c$ for each GaN:Er film can be determined from the XRD data and is listed in the Table I. The GaN:Er film grown on the c-GaN bulk substrate shows almost no strain. However, the lattice constants of the $\mathrm{GaN}$ :Er films grown on other substrates are elongated. Based on the XRD data, the out-of-plane strain parameter $\varepsilon_{\mathrm{c}}$ can be determined from ${ }^{18-20}$

$$
\varepsilon_{\mathrm{c}}=\frac{c-c_{0}}{c_{0}},
$$

where $c_{0}$ is the unstrained lattice constant of $\mathrm{GaN}\left(c_{0}\right.$ $=5.185 \AA$ ) and $c$ is the lattice constant of the samples under strain. ${ }^{20}$ Positive $\varepsilon_{\mathrm{c}}$ means that the thin film exhibits a tensile strain, while negative $\varepsilon_{\mathrm{c}}$ refers to the compressive strain. With the exception of GaN:Er grown on c-GaN bulk, each sample showed a tensile out-of-plane strain in the c-direction which was related to the lattice mismatch between the $\mathrm{GaN}$ :Er epilayer and the template as well to the Er doping. Thin films grown on the lattice-mismatched substrates are under biaxial stress condition. ${ }^{20-22}$ The in-plane strain parameter $\varepsilon_{\mathrm{a}}$ can be expressed as

$$
\varepsilon_{\mathrm{a}}=-\frac{C_{33}}{2 C_{13}} \varepsilon_{\mathrm{c}},
$$

and the biaxial stress $\delta$ can be represented as

TABLE I. The parameters for GaN:Er layers grown on different templates: lattice constant $c$, FWHM of (002) rocking curve, biaxial stress $\delta$, and $I_{\mathrm{emi}}$ at $1.54 \mu \mathrm{m}(T=300 \mathrm{~K})$.

\begin{tabular}{lcccc}
\hline \hline \multicolumn{1}{c}{ Template } & $\begin{array}{c}c \\
(\AA)\end{array}$ & $\begin{array}{c}\text { FWHM } \\
(\operatorname{arcsec})\end{array}$ & $\begin{array}{c}\delta \\
(\mathrm{GPa})\end{array}$ & $\begin{array}{c}I_{\mathrm{emi}} \text { at } 1.54 \mu \mathrm{m} \\
(\text { a.u. })\end{array}$ \\
\hline $\mathrm{AlN} / \mathrm{Al}_{2} \mathrm{O}_{3}$ & 5.195 & 1780 & 1.7 & 33 \\
$\mathrm{GaN} / \mathrm{Al}_{2} \mathrm{O}_{3}$ & 5.189 & 380 & 0.7 & 31 \\
$\mathrm{GaN} / \mathrm{Si}(111)$ & 5.188 & 900 & 0.5 & 24 \\
c-bulk $\mathrm{GaN}$ & 5.185 & 260 & 0 & 8 \\
\hline \hline
\end{tabular}

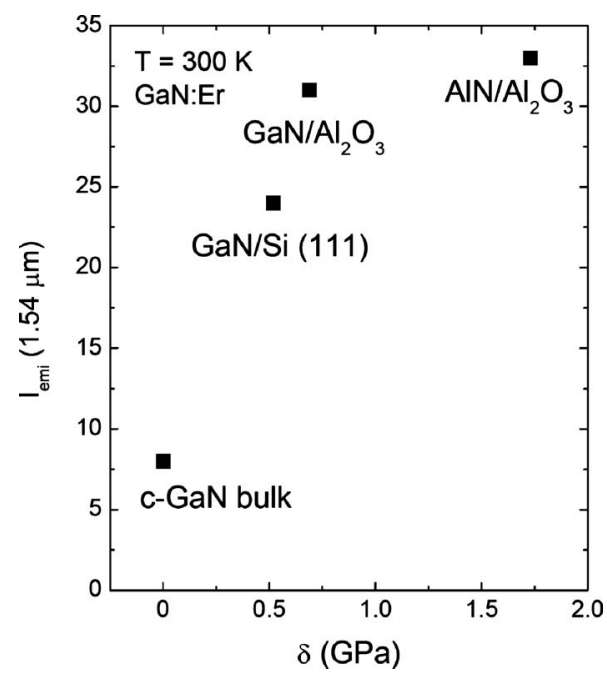

FIG. 3. PL intensity of $1.54 \mu \mathrm{m}$ emission vs the biaxial stress $\delta$ of GaN:Er layers grown on different templates.

$$
\begin{aligned}
& \delta=M_{\mathrm{f}} \varepsilon_{\mathrm{a}}, \\
& M_{\mathrm{f}}=C_{11}+C_{12}-2 \frac{C_{13}^{2}}{C_{33}},
\end{aligned}
$$

where $M_{\mathrm{f}}$ is the biaxial elastic modulus and $C_{\mathrm{ij}}$ are the elastic constants of GaN. Using the reported values of $C_{\mathrm{ij}}\left(C_{11}\right.$ $=390 \mathrm{GPa}, C_{12}=145 \mathrm{GPa}, C_{13}=106 \mathrm{GPa}, \quad$ and $C_{33}$ $=398 \mathrm{GPa}),{ }^{20}$ we have calculated the biaxial stress for these samples. The stress ranges from $0.5 \mathrm{GPa}[\mathrm{GaN}: \mathrm{Er}$ on $\mathrm{GaN} / \mathrm{Si}$ (111)] to $1.7 \mathrm{GPa}\left(\mathrm{GaN}: \mathrm{Er}\right.$ on $\left.\mathrm{AlN} / \mathrm{Al}_{2} \mathrm{O}_{3}\right)$, as summarized in Table I. There is no measurable stress for GaN:Er grown on bulk c-GaN. Furthermore, the XRD spectra as shown in Fig. 2 reveal that GaN:Er grown on $\mathrm{AlN} / \mathrm{Al}_{2} \mathrm{O}_{3}$ has the lowest crystalline quality, while the epilayer grown on $\mathrm{c}-\mathrm{GaN}$ bulk possesses the highest crystalline quality. The calculated values are also consistent with the full width of half maximum (FWHM) data of the XRD spectra listed in Table I. The relatively poorer crystalline quality of $\mathrm{GaN}$ :Er grown on $\mathrm{AlN} / \mathrm{Al}_{2} \mathrm{O}_{3}$ can be attributed to point-defects or dislocations induced by a larger lattice-mismatch between the template and $\mathrm{GaN}$ :Er layer. Although the large stress in the GaN:Er layer grown on $\mathrm{AlN} / \mathrm{Al}_{2} \mathrm{O}_{3}$ template results in poorer crystalline quality, the layer emits the strongest $1.54 \mu \mathrm{m}$ PL intensity. This indicates that the crystalline quality of GaN:Er may not be the vital parameter for optimizing the $1.54 \mu \mathrm{m}$ emission intensity of these Er doped thin films.

Figure 3 shows the PL intensity at $1.54 \mu \mathrm{m}$ as a function of the biaxial stress $\delta$ of GaN:Er layers. By changing the templates from c-GaN bulk to $\mathrm{AlN} / \mathrm{Al}_{2} \mathrm{O}_{3}$, the stress of the thin film increases and the intensity of $1.54 \mu \mathrm{m}$ emission is enhanced roughly by three times. However, the crystalline quality of $\mathrm{GaN}$ :Er grown on $\mathrm{c}-\mathrm{GaN}$ bulk substrate is much better than that of GaN:Er grown on nonlattice matched substrates. The $1.54 \mu \mathrm{m}$ emission intensity increases linearly with the biaxial stress $\delta$ from 0 to $0.7 \mathrm{GPa}$ and reaches saturation for $\mathrm{GaN}: \mathrm{Er}$ grown on $\mathrm{AlN} / \mathrm{Al}_{2} \mathrm{O}_{3}$ templates $(\delta$ $=1.7 \mathrm{GPa}$ ). A similar relationship between $1.54 \mu \mathrm{m}$ emission intensity and strain/stress was also reported for $\mathrm{Si}: \mathrm{Er}$ $+\mathrm{O}$ and $\mathrm{Si}_{x} \mathrm{Ge}_{1-x}$ : $\mathrm{Er}+\mathrm{O}$ materials grown on $\mathrm{Si}$ substrates. ${ }^{4}$ Whether under tensile or compressive stress, strained $\mathrm{Si}$ : Er 
$+\mathrm{O}$ has a stronger $1.54 \mu \mathrm{m}$ emission than an unstrained film.

The correlation between $1.54 \mu \mathrm{m}$ emission and the stress introduced by the lattice-mismatch, shown in Fig. 3, opens up the possibility of engineering GaN:Er photonic devices with enhanced emission intensity at $1.54 \mu \mathrm{m}$. In the free ion $\mathrm{Er}^{3+}$ state, these electric dipole transitions $\left({ }^{4} \mathrm{I}_{13 / 2}\right.$ $\left.-{ }^{4} \mathrm{I}_{15 / 2}\right)$ are forbidden to first order because of parity conservation. Within the GaN host, local electric fields produce a Stark splitting of the energy levels of the $\mathrm{Er}^{3+}$ ions and certain $4 f$ transitions are possible. Since the wurtzite GaN structure has no inversion symmetry, there is an internal electric field due to spontaneous polarization. Furthermore, stress from the lattice-mismatch produces an additional electric field as a result of the piezoelectric effect. The electric fields acting on the $\mathrm{Er}^{3+}$ ions lift the degeneracy of the $4 f$ energy levels and lead to emission at $1.54 \mu \mathrm{m}$. Consequently, the current experiments show that enhanced emission intensity at $1.54 \mu \mathrm{m}$ is possible through strain engineering.

In summary, the optical characteristics and crystalline quality of GaN:Er samples grown on different templates were studied. The measured PL emission at $1.54 \mu \mathrm{m}$ was stronger in $\mathrm{GaN}: \mathrm{Er}$ epilayers grown on $\mathrm{GaN} / \mathrm{Si}$ (111) than in those grown on c-bulk GaN, regardless of the crystalline quality and surface morphology. The XRD data indicate that the $1.54 \mu \mathrm{m}$ emission intensity increases as stress of GaN:Er layer increases. These results demonstrate that GaN:Er layers grown by MOCVD on Si substrates may provide a basis for efficient RT photonic devices.

The MOCVD growth effort is supported by ARO (Grant No. W911NF-09-1-0275) and U.S. Army CERDEC (Grant No. W15P7T-07-D-P040) and PL study effort is supported by NSF (Grant No. ECCS-0854619). H.X.J. and J.Y.L. would like to acknowledge the support of Whitacre endowed chairs through AT\&T foundation.
${ }^{1}$ S. Chen, J. Seo, J. Sawahata, and K. Akimoto, J. Cryst. Growth 311, 2042 (2009)

${ }^{2}$ C. Ugolini, N. Nepal, J. Y. Lin, H. X. Jiang, and J. M. Zavada, Appl. Phys. Lett. 89, 151903 (2006).

${ }^{3}$ M. R. Correia, S. Pereira, A. Cavaco, and E. Pereira, Appl. Phys. Lett. 80, 4504 (2002).

${ }^{4}$ T. Ishiyama, S. Yoneyama, Y. Yamashita, Y. Kamiura, T. Date, T. Hasegawa, K. Inoue, and K. Okuno, Physica B 376, 122 (2006).

${ }^{5}$ P. H. Citrin, P. A. Northrup, R. Birkhahn, and A. J. Steckl, IEEE proceedings of 2000 International Semiconducting and Insulating Materials Conference (SIMC-XI), 2000 (unpublished), pp. 15-22.

${ }^{6}$ T. D. Culp, U. Hömmerich, J. M. Redwing, T. F. Kuech, and K. L. Bray, J. Appl. Phys. 82, 368 (1997).

${ }^{7}$ J. M. Redwing, T. F. Kuech, D. C. Gordon, B. A. Vaartstra, and S. S. Lau, J. Appl. Phys. 76, 1585 (1994).

${ }^{8}$ Y. Fujiwara, N. Matsubara, J. Tsuchiya, T. Ito, and Y. Takeda, Jpn. J. Appl. Phys., Part 1 36, 2587 (1997).

${ }^{9}$ P. N. Favennec, H. L'Haridon, M. Salvi, D. Moutonnet, and Y. Le Guillou, Electron. Lett. 25, 718 (1989).

${ }^{10} \mathrm{~V}$. Glukhanyuk, H. Przybylinska, A. Kozanecki, and W. Jantsch, Opt. Mater. 28, 746 (2006).

${ }^{11}$ K. Makarova, M. Stachowicz, V. Glukhanyuk, A. Kozanecki, C. Ugolini, J. Y. Lin, H. X. Jiang, and J. M. Zavada, Mater. Sci. Eng., B 146, 193 (2008).

${ }^{12}$ R. H. Birkhahn, R. Hudgins, D. S. Lee, B. K. Lee, A. J. Steckl, A. Saleh, R. G. Wison, and J. M. Zavada, MRS Internet J. Nitride Semicond. Res. 4S1, G3.80 (1999).

${ }^{13}$ D. S. Lee, J. Heikenfeld, and A. J. Steckl, Appl. Phys. Lett. 80, 344 (2002).

${ }^{14}$ R. Birkhahn, R. Hudgins, D. Lee, A. J. Steckl, R. J. Molnar, and J. M. Zavada, J. Vac. Sci. Technol. B 17, 1195 (1999).

${ }^{15}$ C. Ugolini, N. Nepal, J. Y. Lin, H. X. Jiang, and J. M. Zavada, Appl. Phys. Lett. 90, 051110 (2007).

${ }^{16}$ R. Dahal, C. Ugolini, J. Y. Lin, H. X. Jiang, and J. M. Zavada, Appl. Phys. Lett. 93, 033502 (2008).

${ }^{17}$ R. Dahal, C. Ugolini, J. Y. Lin, H. X. Jiang, and J. M. Zavada, Appl. Phys. Lett. 95, 111109 (2009).

${ }^{18}$ A. F. Wright, J. Appl. Phys. 82, 2833 (1997).

${ }^{19}$ S. I. Cho, K. Chang, and M. S. Kwon, J. Mater. Sci. 42, 3569 (2007).

${ }^{20}$ V. S. Harutyunyan, A. P. Aivazyan, E. R. Weber, Y. Kim, Y. Park, and S. G. Subramanya, J. Phys. D 34, A35 (2001).

${ }^{21}$ B. N. Pantha, N. Nepal, T. M. Al Tahtamouni, M. L. Kakarmi, J. Li, J. Y. Lin, and H. X. Jiang, Appl. Phys. Lett. 91, 121117 (2007).

${ }^{22}$ K. Jeganathan, M. Shimizu, and H. Okumura, J. Appl. Phys. 97, 013524 (2005) 